\title{
Conversion from chronic to episodic migraine in patients treated with erenumab: real-life data from an Italian region
}

Raffaele Ornello ${ }^{1}$, Alfonsina Casalena ${ }^{2}$, Ilaria Frattale ${ }^{1}$, Valeria Caponnetto ${ }^{1}$, Amleto Gabriele ${ }^{3}$, Giannapia Affaitati ${ }^{4}$, Maria Adele Giamberardino ${ }^{4}$, Maurizio Assetta ${ }^{2}$, Maurizio Maddestra ${ }^{5}$, Fabio Marzoli ${ }^{5}$, Stefano Viola ${ }^{6}$,

Davide Cerone ${ }^{7}$, Carmine Marini ${ }^{8}$, Francesca Pistoia ${ }^{1,7}$ and Simona Sacco ${ }^{1 *}$ (I)

\begin{abstract}
Background: Most patients treated with erenumab in clinical practice have chronic migraine (CM). We assessed the rate and possible predictors of conversion from CM to episodic migraine (EM) in a real-life study.

Main body: We performed a subgroup analysis of patients treated with erenumab from January 2019 to February 2020 in the Abruzzo region, central Italy. Treatment was provided according to current clinical practice. For the purpose of the present study, we included patients fulfilling the definition of CM for the three months preceding erenumab treatment and with at least 6 months of follow-up after treatment. We assessed the rate of conversion to EM from baseline to Months 4-6 of treatment and during each month of treatment. To test the clinical validity of conversion to EM, we also assessed the decrease in monthly headache days (MHDs), acute medication days, and median headache intensity on a Numerical Rating Scale (NRS). We included in our study 91 patients with CM. At Months 4-6, 62 patients (68.1\%) converted from CM to EM; the proportion of converters increased from Month 1 to Month 5. In the overall group of patients, median MHDs decreased from 26.5 (IQR 20-30) to 7.5 (IQR 5-16; P< 0.001) compared with baseline, while median acute medication days decreased from 21 (IQR 16-30) to 6 (IQR 3-10; $P<0.001$ ) and median NRS scores decreased from 8 (IQR 7-9) to 6 (IQR 4-7; $P<0.001$ ). Significant decreases were found both in converters and in non-converters. We found no significant predictors of conversion to EM among the patients' baseline characteristics.
\end{abstract}

Conclusions: In our study, two thirds of patients with CM converted to EM during 6 months of treatment with erenumab. MHDs, acute medication use, and headache intensity decreased regardless of conversion from CM to EM.

Keywords: Chronic migraine, Calcitonin gene-related peptide, Migraine prevention, Monoclonal antibodies, Erenumab, Real-life study

\footnotetext{
* Correspondence: simona.sacco@univaq.it

'Department of Applied Clinical Sciences and Biotechnology, University of L'Aquila, Via Vetoio 1, 67100 L'Aquila, Italy

Full list of author information is available at the end of the article
}

(c) The Author(s). 2020 Open Access This article is licensed under a Creative Commons Attribution 4.0 International License, which permits use, sharing, adaptation, distribution and reproduction in any medium or format, as long as you give appropriate credit to the original author(s) and the source, provide a link to the Creative Commons licence, and indicate if changes were made. The images or other third party material in this article are included in the article's Creative Commons licence, unless indicated otherwise in a credit line to the material. If material is not included in the article's Creative Commons licence and your intended use is not permitted by statutory regulation or exceeds the permitted use, you will need to obtain permission directly from the copyright holder. To view a copy of this licence, visit http://creativecommons.org/licenses/by/4.0/. The Creative Commons Public Domain Dedication waiver (http://creativecommons.org/publicdomain/zero/1.0/) applies to the data made available in this article, unless otherwise stated in a credit line to the data. 


\section{Background}

Migraine can be classified as episodic (EM) or chronic (CM) according to the number of monthly headache days (MHDs) [1]. CM carries a high burden of disability as it is often associated with medication overuse [2]; besides, patients are often unaware of the availability of effective treatment options [3, 4]. Monoclonal antibodies directed against the calcitonin gene-related peptide (CGRP) or its receptor (CGRPr) are migraine-specific treatments whose efficacy and safety were proven in both EM and CM [5-8]. However, the available real-life studies show that most patients treated in common clinical practice have CM [9-13]. In the present real-life, multicenter study, we assessed the rate and possible predictors of conversion from CM to EM in patients treated with erenumab.

\section{Methods}

\section{Study population}

We performed a subgroup analysis of a real-life study [10]. We included patients aged 18 to 65 years consecutively treated with erenumab in the Headache Centers of Avezzano, L'Aquila, Sulmona, Teramo, Chieti, Lanciano, and Vasto, all located in the Abruzzo region, central Italy, from January 2019 to February 2020. The study was approved by the Internal review Board of the University of L'Aquila with the number 44/2019. All patients signed an informed consent.

Erenumab was provided to patients from the producing company upon reasonable request from the Headache Centers. The drug was provided for patients with migraine with or without aura diagnosed by expert physicians according to the International Classification of Headache Disorders (ICHD-3) criteria [1]. Subjects were selected for treatment if having $\geq 4$ MHDs and $\geq 2$ prior preventive treatment failures, according to the European Headache Federation [5] and the American Headache Society [14] criteria.

For the purpose of the present study, we only included patients fulfilling the ICHD-3 criteria for $\mathrm{CM}$ at baseline. We excluded from the present study patients with EM and patients with history of $\mathrm{CM}$ that had converted to $\mathrm{EM}$ in the three months preceding the treatment. The resulting study population was of 91 patients with CM.

\section{Treatment procedure}

Erenumab was administered during in-person visits in a monthly subcutaneous dose of $70 \mathrm{mg}$, with the option of switching to $140 \mathrm{mg}$ monthly in case of a $<30 \%$ decrease in MHDs compared with baseline; patients with several prior preventive treatment failures could start treatment with a $140 \mathrm{mg}$ monthly dose according to the treating physician's judgement. The treatment was continued at least until Month 6, but we acknowledged the possibility of early withdrawal because of severe adverse events, lack of compliance, or ineffectiveness $(<30 \%$ reduction in MHDs and/or lack of satisfaction with treatment). All patients, including those who stopped treatment, were followed-up for 6 months. As this was an observational real-life study, during the period of erenumab treatment the patients were allowed to start, continue, or discontinue concurrent oral preventive treatments for migraine according to clinical indication and also considering patients' preferences. Patients with medication overuse were not detoxified prior to erenumab treatment, according to current recommendations [5].

\section{Data collection}

For each included patient, we recorded sex, age, migraine and $\mathrm{CM}$ duration, migraine frequency and intensity, acute and preventive treatments as reported in the patients' headache diaries. We assessed attack severity by the $0-10$ Numerical Rating Scale (NRS). We also assessed the presence of risk factors for $\mathrm{CM}$, including obesity, sleep disturbances, and depressive symptoms. Obesity was defined as Body Mass Index value $\geq 30 \mathrm{~kg} /$ $\mathrm{m}^{2}$; sleep disturbances were defined as any patient report, while depressive symptoms were defined as a Beck Depression Inventory score $\geq 20$ or use of antidepressant medications not prescribed for migraine. Data were collected with a clinical interview and then reported on a standardized form with pre-determined answers which was the same for all participating centers. All the recorded data were stored in an anonymized computerized database.

\section{Statistical analysis}

Data from all patients receiving at least one erenumab dose were included in the analyses. Baseline was defined as the monthly mean of the three months preceding erenumab treatment. 'Converters' were defined as subjects fulfilling the definition of EM at Months 4-6 of treatment, while the remaining patients, including those discontinuing erenumab treatment, were defined as 'nonconverters'. Calculations at Months 4-6 were based on the mean values of the three months. The primary outcome of the study was the overall rate of converters at Months 4-6; the secondary outcomes of the study were to evaluate the rate of converters per each month of treatment from Month 1 to Month 6 and the rates of high-frequency (HFEM; 8-14 MHDs), mediumfrequency (MFEM; 4-7 MHDs), and low-frequency EM (LFEM; 0-3 MHDs) at Months 4-6 and per each month of treatment. To verify the clinical validity of assessing $\mathrm{CM}$ conversion, we compared the treatment outcomes (decrease in MHDs, days of acute medication, and median NRS from baseline to Months 4-6) in converters 
Table 1 Characteristics of the study patients

\begin{tabular}{ll}
\hline Characteristics (total patients =91) & \\
\hline Female, $n$ (\%) & $80(87.9)$ \\
Age, median (IQR) & $49(39-54)$ \\
Years of migraine history, median (IQR) & $28.5(20-34)$ \\
Years of CM history, median (IQR) & $10(4-19)$ \\
Baseline MHDs, median (IQR) & $26.5(20-30)$ \\
Baseline acute medication days, median (IQR) & $21(16-30)$ \\
Baseline NRS, median (IQR) & $8(7-9)$ \\
Aura, $n$ (\%) & $28(30.8)$ \\
Allodynia, $n$ (\%) & $35(38.5)$ \\
Medication overuse, $n$ (\%) & $71(78.0)$ \\
Previous preventive treatment failures, $n$ (\%) & \\
2 & $31(43.1)$ \\
3 & $24(26.4)$ \\
4 & $28(30.8)$ \\
$>4$ & $8(8.8)$ \\
Botulinum toxin failure, $n$ (\%) & $39(42.9)$ \\
Concurrent oral preventive treatments at baseline, $n(\%)$ & $30(33.0)$ \\
Obesity, $n$ (\%) & $13(14.3)$ \\
Sleep disturbances, $n(\%)$ & $33(36.3)$ \\
Depressive symptoms, $n$ (\%) & $19(20.9)$ \\
\hline
\end{tabular}

$C M$ indicates chronic migraine, IQR interquartile range, $M H D$ monthly headache days, NRS Numerical Rating Scale

and in non-converters. Assessment timepoints were chosen in accordance to the STRIVE trial [15].

Categorical data were reported as numbers and proportions, while continuous data were reported as medians and interquartile ranges (IQRs). We used the chi- square test to compare categorical variables and the Mann-Whitney U test to compare medians. Statistical significance was set at $P<0.05$. We estimated that a sample size of 43 subjects would be adequate to detect a $68 \%$ conversion rate, as previously reported [16], with a 95\% confidence interval and 90\% precision.

\section{Results}

All the 91 patients with CM were followed-up for at least six months in the absence of losses to follow-up; 11 patients $(12.1 \%)$ discontinued the treatment before Month 6 due to ineffectiveness (10 patients) or adverse events ( 1 patient). Table 1 reports the baseline characteristics of the study patients.

Sixty-two $(68.1 \%)$ patients were converters at Months 4-6. Monthly converters increased from 44 (48.4\%) at Month 1 to 65 (71.4\%) at Month 5 (Fig. 1). At Months 4-6, 15 (16.5\%) patients achieved the status of LFEM, 26 (28.6\%) MFEM, and 21 (23.1\%) HFEM. Figure 1 shows the proportion of patients with LFEM, MFEM, and HFEM after each month of treatment. Thirty-eight $(41.8 \%)$ patients reached the converter status without needing erenumab dose increase from $70 \mathrm{mg}$ to $140 \mathrm{mg}$ monthly, while $24(26.4 \%)$ patients needed a dose increase; all non-converters increased the erenumab dose during follow-up. Concurrent migraine preventive treatments were discontinued in 11 (12.1\%) patients.

At Months 4-6, median MHDs decreased from 26.5 (IQR 20-30) to 7.5 (IQR 5-16; P< 0.001) compared with baseline in the overall group, from 25 (IQR 20-30) to 6 (IQR 3-8; $P<0.001$ ) in converters, and from 30 (IQR $20-30$ ) to 20.5 (IQR $17.5-28.5 ; P=0.003$ ) in nonconverters (Fig. 2-a). Median acute medication days

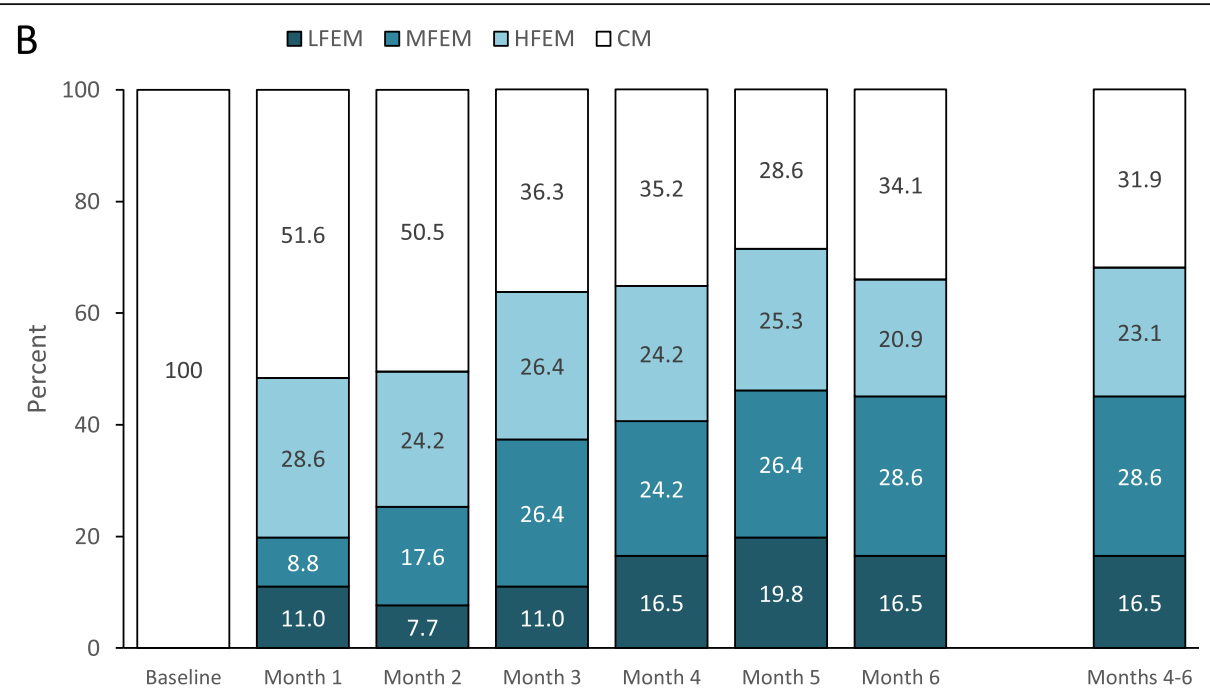

Fig. 1 Rates of conversion to episodic migraine at Months 4-6 and after each month of treatment according to monthly headache days. HFEM indicates high-frequency episodic migraine (8-14 monthly headache days); LFEM, low-frequency episodic migraine (0-3 monthly headache days); MFEM, medium-frequency episodic migraine (4-7 monthly headache days) 


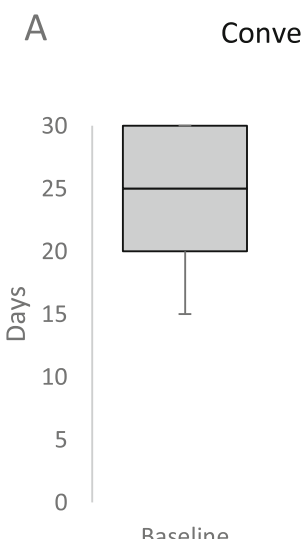

Baseline

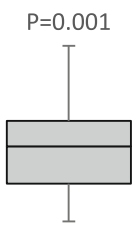

Months 4-6

\section{B}

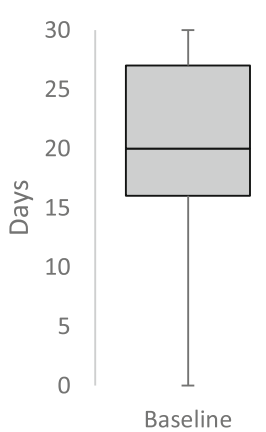

C

Converters

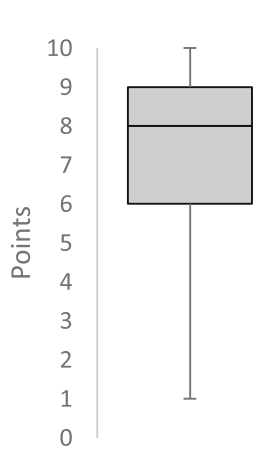

Baseline

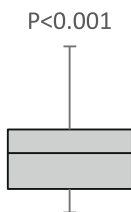

Months 4-6

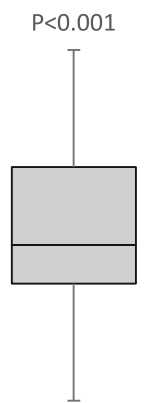

Months 4-6
Non-converters

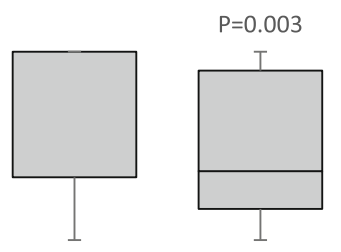

Baseline

Months 4-6

Non-converters

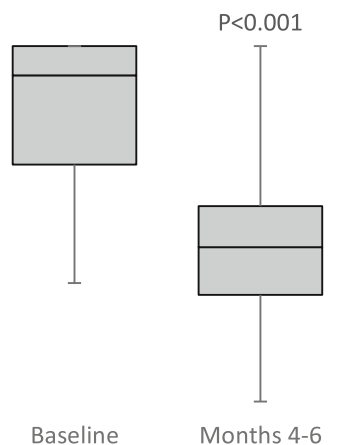

Non-converters

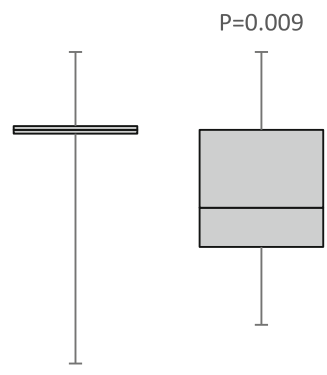

Baseline

Months 4-6

Fig. 2 Decrease in median headache days $\mathbf{a}$, acute medication days $\mathbf{b}$, and headache intensity on a Numerical Rating Scale $\mathbf{c}$ according to converter status in the present study

decreased from 21 (IQR 16-30) to 6 (IQR 3-10; $P<$ 0.001 ) in the overall group, from 20 (IQR 16-27) to 5 (IQR 2-7; $P<0.001$ ) in converters, and from 27.5 (IQR $20-30$ ) to 13 (IQR 9-16.5; $P<0.001$ ) in non-converters (Fig. 2b). In detail, median days of triptan use changed from 4 (IQR $0-20$ ) to 2 (IQR $0-6 ; P<0.001$ ) in the overall group, from 6 (IQR $0-20$ ) to 1 (IQR $0-5 ; P<$ 0.001 ) in converters, and from 0 (IQR $0-15$ ) to 3 (IQR $0-8 ; P=0.043)$ in non-converters, while median days of common analgesic use decreased from 11 (IQR 4-25) to 3 (IQR 1-6; $P<0.001$ ) in the overall group, from 8 (IQR $0-20)$ to 1 (IQR $0-4 ; P<0.001$ ) in converters, and from 
20 (IQR $10-30$ ) to 11 (IQR 6-18; $P=0.002$ ) in nonconverters. Median NRS decreased from 8 (IQR 7-9) to 6 (IQR 4-7; $P<0.001$ ) in the overall group, from 8 (IQR $6-9)$ to 5 (IQR $4-7 ; P<0.001$ ) in converters, and from 8 (IQR 8-8) to 6 (IQR 5-8; $P=0.009$ ) in non-converters (Fig. 2c). All the 46 converters and 16 (64.0\%) of the 25 non-converters with medication overuse withdrew from that condition.

Univariate comparisons showed no differences in baseline characteristics between converters and nonconverters (Table 2).

\section{Discussion}

Our data show that two thirds of patients with CM convert to EM during a 6-month treatment with erenumab. The proportion of patients converting to EM was about half at Month 1 and increased up to three quarters at Month 5. All converters withhold medication overuse. The high rate of conversion to EM in our population of difficult-to-treat patients with a long history of CM and multiple prior preventive treatment failures, including botulinum toxin in $\geq 40 \%$ of cases, supports the efficacy of erenumab for the preventive treatment of patients with $\mathrm{CM}$, as shown in randomized controlled trials [1722] and real-life studies [10-13]. We also found that at Months 4-6 16.5\% of patients achieved a status of LFEM, while $28.6 \%$ achieved a status of MFEM, which indicates a high treatment benefit and a substantial improvement in the patients' quality of life.
Notably, the treatment decreased headache frequency, intensity, and use of triptans and common analgesics in both converters and non-converters, suggesting that even patients who do not convert to EM may have benefits from erenumab treatment. Erenumab treatment also had a relevant effect on medication overuse withdrawal both in converters and in non-converters. With regard to those findings, it should be noted that CM and EM are not distinct entities, as suggested by the frequent fluctuations between the two conditions [23] and the similar levels of disability associated with $\mathrm{CM}$ and HFEM [24].

We found no predictors of conversion to EM, even when considering characteristics associated with $\mathrm{CM}$ such as sleep disturbances, obesity, and depressive symptoms [25]. Further larger studies are needed to assess reliable predictors of favorable response to anti-CGRP treatment in order to maximize treatment outcomes.

We collected a sample of patients treated according to clinical practice in a multicenter study. Besides, to ensure reliable statistical estimates, we chose to perform nonparametric tests such as the Wilcoxon test. However, our study also suffers from several limitations. Due to low numbers, we could not assess the role of concurrent migraine preventive treatments and of several factors influencing migraine, such as seasonality or life events. We also could not assess the added contribution of escalating erenumab dose from $70 \mathrm{mg}$ to $140 \mathrm{mg}$ monthly, which might have been relevant in patients with multiple prior preventive treatment failures [26]. Besides, we did

Table 2 Baseline characteristics of converters versus non-converters to episodic migraine during the treatment

\begin{tabular}{|c|c|c|c|}
\hline Characteristic & Converters $(n=62)$ & Non-converters $(n=29)$ & $P$ value \\
\hline Female, $n(\%)$ & $54(87.1)$ & $26(89.7)$ & 0.727 \\
\hline Age, median (IQR) & $47(38-51)$ & $53(42-57)$ & 0.060 \\
\hline Years of migraine history, median (IQR) & $28(20-33)$ & $29(20-37)$ & 0.435 \\
\hline Years of CM history, median (IQR) & $8(5-12)$ & $15(4-22)$ & 0.099 \\
\hline MHDs, median (IQR) & $25(20-30)$ & $30(20-30)$ & 0.360 \\
\hline Acute medication days, median (IQR) & $20(16-27)$ & $27.5(20-30)$ & 0.063 \\
\hline Baseline NRS, median (IQR) & $8(6-9)$ & $8(8-8)$ & 0.349 \\
\hline Aura, $n(\%)$ & $18(29.0)$ & $10(34.4)$ & 0.600 \\
\hline Allodynia, $n(\%)$ & $24(38.7)$ & $11(37.9)$ & 0.943 \\
\hline Medication overuse, $n$ (\%) & $46(74.2)$ & $25(86.2)$ & 0.197 \\
\hline Prior preventive treatment failures, $n(\%)$ & & & 0.954 \\
\hline 2 & $21(33.9)$ & $10(34.5)$ & \\
\hline$>2$ & $41(66.1)$ & $19(65.5)$ & \\
\hline Botulinum toxin failure, $n(\%)$ & $26(41.9)$ & $13(44.8)$ & 0.795 \\
\hline Obesity, n (\%) & $9(14.5)$ & $4(13.8)$ & 0.999 \\
\hline Sleep disturbances, $n$ (\%) & 19 (30.6) & $14(48.3)$ & 0.103 \\
\hline Depressive symptoms, n (\%) & $13(21.0)$ & $6(20.7)$ & 0.976 \\
\hline
\end{tabular}

CM indicates chronic migraine, IQR interquartile range, MHD monthly headache days, NRS Numerical Rating Scale 
not have data on patient-reported outcomes, such as quality of life measures, which would have been useful to better quantify the benefit of erenumab. Better measures are needed to exactly quantify the response to anti-CGRP migraine preventive treatments and to identify factors that can predict good treatment outcomes. Lastly, we cannot exclude that in some patients the conversion to EM was due to the natural fluctuation of the disease or to placebo effect rather than to the treatment itself.

\section{Conclusion}

In our study, two thirds of patients with $\mathrm{CM}$ converted to EM during 6 months of treatment with erenumab. $\mathrm{Pa}$ tients reported a decrease in MHDs, headache intensity, and acute medication use irrespective of their converter status.

\section{Abbreviations}

CGRPr: Calcitonin gene-related peptide receptor; CM: Chronic migraine; EM: Episodic migraine; HFEM: High-frequency episodic migraine; ICHD3: International Classification of Headache Disorders, 3rd Edition; LFEM: Lowfrequency episodic migraine; MFEM: Medium-frequency episodic migraine; MHDs: Monthly headache days; NRS: Numerical Rating Scale

\section{Acknowledgements}

The Authors wish to thank all the study patients for their kind cooperation.

\section{Authors' contributions}

$\mathrm{RO}$ and SS conceived the study and its design, performed the acquisition, analysis, interpretation of data, and drafted the manuscript. CT, IF, GP, CM, and FP substantively revised the manuscript. All Authors approved the final manuscript.

\section{Funding}

The publication fee for the present paper was unconditionally granted by Novartis Farma S.r.l. Prof. Francesca Pistoia states that the study was not sponsored by Novartis Farma S.r.l., which only contributed to cover publication fees.

\section{Availability of data and materials}

Anonymized data operated or analyzed during this study are available from the Authors upon reasonable request.

\section{Ethics approval and consent to participate}

The study was approved by the Internal Review Board of the University of L'Aquila (Italy) and patients gave written informed consent according to the Declaration of Helsinki.

\section{Consent for publication}

Not applicable.

\section{Competing interests}

RO has received sponsorship to attend meetings from Novartis and Teva; SS had a financial relationship (lecturer or member of advisory board) with Abbott, Allergan, Novartis, Teva, and Eli Lilly; GA has received funds for congress participation from Innovet Italia Srl, Epitech Group and Lusofarmaco; MAG received funds for congress participation from IBSA; AC, $I F, A G, M A, M M, F M, S V, D C, C M$, and FP declare no competing interests.

\section{Author details}

'Department of Applied Clinical Sciences and Biotechnology, University of L'Aquila, Via Vetoio 1, 67100 L'Aquila, Italy. ${ }^{2}$ Department of Neurology, 'G. Mazzini' Hospital, Teramo, Italy. ${ }^{3}$ Neurology Service, 'SS. Annunziata' Hospital, Sulmona, Italy. ${ }^{4}$ Department of Medicine and Science of Aging, 'G. D'Annunzio' University, Chieti, Italy. ${ }^{5}$ Department of Neurology, 'F. Renzetti'
Hospital, Lanciano, Italy. ${ }^{6}$ Department of Neurology, 'S. Pio da Pietrelcina' Hospital, Vasto, Italy. 'Department of Neurology, 'S. Salvatore' Hospital, L'Aquila, Italy. ${ }^{8}$ Department of Medicine, Public Health, Life and Environmental Sciences, University of L'Aquila, L'Aquila, Italy.

Received: 19 July 2020 Accepted: 11 August 2020

Published online: 15 August 2020

\section{References}

1. Headache Classification Committee of the International Headache Society (IHS) The International Classification of Headache Disorders, 3rd edition. Cephalalgia. 2018;38 1:1-211; doi: https://doi.org/10.1177/ 0333102417738202

2. Del Valle López Martínez M, Pareja Román J, Jiménez Hernández MDU, Ceferino del Carmen Ramírez-Castillejo, María. Chronic Migraine with Medication Overuse: Clinical Pattern and Evolution from a Retrospective Cohort in Seville, Spain. SN Comprehensive Clinical Medicine. 2020; doi: https://doi.org/10.1007/s42399-020-00424-8

3. Goodhew SC (2019) Migraine literacy and treatment in a university sample. SN Comp Clin Med 1:749-757. https://doi.org/10.1007/s42399-019-00124-y

4. Ali MW, Musami UB, Sa'ad FK, Omoaghe C, Danimoh MA, Ayoola YA et al (2020) Profile of migraine patients in a developing country: a multicentre study. SN Comp Clin Med. https://doi.org/10.1007/s42399-020-00394-x

5. Sacco S, Bendtsen L, Ashina M, Reuter U, Terwindt G, Mitsikostas DD et al (2019) European headache federation guideline on the use of monoclonal antibodies acting on the calcitonin gene related peptide or its receptor for migraine prevention. J Headache Pain 20(1):6. https://doi.org/10.1186/ s10194-018-0955-y

6. Tiseo C, Ornello R, Pistoia F, Sacco S (J Headache Pain, 2019) How to integrate monoclonal antibodies targeting the calcitonin gene-related peptide or its receptor in daily clinical practice. 20(1):49. https://doi.org/10. 1186/s10194-019-1000-5

7. Negro A, Martelletti P (2019) Patient selection for migraine preventive treatment with anti-CGRP(r) monoclonal antibodies. Expert Rev Neurother 19(8):769-776. https://doi.org/10.1080/14737175.2019.1621749

8. De Matteis E, Guglielmetti M, Ornello R, Spuntarelli V, Martelletti P, Sacco S (2020) Targeting CGRP for migraine treatment: mechanisms, antibodies, small molecules, perspectives. Expert Rev Neurother:1-15. https://doi.org/10. 1080/14737175.2020.1772758

9. Barbanti P, Aurilia C, Egeo G, Fofi L (2019) Erenumab: from scientific evidence to clinical practice-the first Italian real-life data. Neurol Sci 40(Suppl 1):177-179. https://doi.org/10.1007/s10072-019-03839-x

10. Ornello R, Casalena A, Frattale I, Gabriele A, Affaitati G, Giamberardino MA et al (2020) Real-life data on the efficacy and safety of erenumab in the Abruzzo region, central Italy. J Headache Pain 21(1):32. https://doi.org/10. 1186/s10194-020-01102-9

11. Russo A, Silvestro M, Scotto di Clemente F, Trojsi F, Bisecco A, Bonavita S et al (2020) Multidimensional assessment of the effects of erenumab in chronic migraine patients with previous unsuccessful preventive treatments: a comprehensive real-world experience. J Headache Pain 21(1):69. https:// doi.org/10.1186/s10194-020-01143-0

12. Lambru G, Hill B, Murphy M, Tylova I, Andreou AP (2020) A prospective realworld analysis of erenumab in refractory chronic migraine. J Headache Pain 21(1):61. https://doi.org/10.1186/s10194-020-01127-0

13. Raffaelli B, Kalantzis R, Mecklenburg J, Overeem LH, Neeb L, Gendolla A et al (2020) Erenumab in chronic migraine patients who previously failed five first-line Oral prophylactics and OnabotulinumtoxinA: a dual-center retrospective observational study. Front Neurol 11:417. https://doi.org/10. 3389/fneur.2020.00417

14. American Headache Society (2019) The American Headache Society Position Statement On Integrating New Migraine Treatments Into Clinical Practice. Headache 59(1):1-18. https://doi.org/10.1111/head.13456

15. Goadsby PJ, Reuter U, Hallström Y, Broessner G, Bonner JH, Zhang F et al (2017) A controlled trial of Erenumab for episodic migraine. N Engl J Med 377(22):2123-2132. https://doi.org/10.1056/NEJMoa1705848

16. Lipton R, Tepper S, Silberstein S, Kudrow D, Ashina M, Reuter U et al (2019) conversion from chronic migraine (CM) to episodic migraine (EM) with long-term Erenumab treatment (S17.008). Neurology. 92

17. Ashina M, Tepper S, Brandes JL, Reuter U, Boudreau G, Dolezil D et al (2018) Efficacy and safety of erenumab (AMG334) in chronic migraine patients with prior preventive treatment failure: a subgroup analysis of a 
randomized, double-blind, placebo-controlled study. Cephalalgia. 38(10): 1611-1621. https://doi.org/10.1177/0333102418788347

18. Brandes JL, Diener HC, Dolezil D, Freeman MC, MCAllister PJ, Winner $P$ et al (2020) The spectrum of response to erenumab in patients with chronic migraine and subgroup analysis of patients achieving $>/=50 \%,>/=75 \%$, and 100\% response. Cephalalgia. 40(1):28-38. https://doi.org/10.1177/ 0333102419894559

19. Lipton RB, Tepper SJ, Reuter U, Silberstein S, Stewart WF, Nilsen J et al (2019) Erenumab in chronic migraine: patient-reported outcomes in a randomized double-blind study. Neurology. 92(19):e2250-e2e60. https://doi. org/10.1212/WNL.00000000000007452

20. Tepper SJ AM, Brandes J, Dolezil D, Silberstein SD, Winner PK, Zhang F, Cheng S, Mikol DD: Assessment of the long-term safety and efficacy of erenumab during open-label treatment of subjects with chronic migraine. In: 12th European headache federation congress jointly with 32nd National Congress of the Italian Society for the Study of headaches. Vol. 19. Florence, Italy: The Journal of Headache and Pain; 2018

21. Tepper S, Ashina M, Reuter U, Brandes JL, Doležil D, Silberstein S et al (2017) Safety and efficacy of erenumab for preventive treatment of chronic migraine: a randomised, double-blind, placebo-controlled phase 2 trial. Lancet Neurol 16(6):425-434. https://doi.org/10.1016/S1474-4422(17)30083-2

22. Tepper SJ, Diener HC, Ashina M, Brandes $J$ L, Friedman DI, Reuter $\mathrm{U}$ et al (2019) Erenumab in chronic migraine with medication overuse: subgroup analysis of a randomized trial. Neurology. 92(20):e2309-e2e20. https://doi. org/10.1212/WNL.0000000000007497

23. Serrano D, Lipton RB, Scher Al, Reed ML, Stewart WBF, Adams AM et al (2017) Fluctuations in episodic and chronic migraine status over the course of 1 year: implications for diagnosis, treatment and clinical trial design. J Headache Pain 18(1):101. https://doi.org/10.1186/s10194-017-0787-1

24. Torres-Ferrus M, Quintana M, Fernandez-Morales J, Alvarez-Sabin J, PozoRosich P (2017) When does chronic migraine strike? A clinical comparison of migraine according to the headache days suffered per month. Cephalalgia. 37(2):104-113. https://doi.org/10.1177/0333102416636055

25. May A, Schulte LH (2016) Chronic migraine: risk factors, mechanisms and treatment. Nat Rev Neurol 12(8):455-464. https://doi.org/10.1038/nrneurol. 2016.93

26. Ornello R, Tiseo C, Frattale I, Perrotta G, Marini C, Pistoia F et al (2019) The appropriate dosing of erenumab for migraine prevention after multiple preventive treatment failures: a critical appraisal. J Headache Pain 20(1):99. https://doi.org/10.1186/s10194-019-1054-4

\section{Publisher's Note}

Springer Nature remains neutral with regard to jurisdictional claims in published maps and institutional affiliations.

Ready to submit your research? Choose BMC and benefit from:

- fast, convenient online submission

- thorough peer review by experienced researchers in your field

- rapid publication on acceptance

- support for research data, including large and complex data types

- gold Open Access which fosters wider collaboration and increased citations

- maximum visibility for your research: over $100 \mathrm{M}$ website views per year

At $\mathrm{BMC}$, research is always in progress.

Learn more biomedcentral.com/submissions 